\title{
Effect of hypoxia on lung gene expression and proteomic profile: insights into the pulmonary surfactant response
}

\author{
Bárbara Olmeda ${ }^{1}$, Todd M. Umstead ${ }^{2}$, Patricia Silveyra ${ }^{2}$, Alberto Pascual ${ }^{3}$, José López- \\ Barneo $^{3}$, David S. Phelps ${ }^{2}$, Joanna Floros ${ }^{2}$, and Jesús Pérez-Gil ${ }^{1}$ \\ ${ }^{1}$ Dept. Bioquímica, Fac. Biología, Universidad Complutense, Madrid, Spain \\ ${ }^{2}$ Center for Host defense, Inflammation, and Lung Disease (CHILD), Department of Pediatrics, \\ The Pennsylvania State University College of Medicine, Hershey, Pennsylvania, USA \\ ${ }^{3}$ Instituto de Biomedicina de Sevilla (IBiS), Hospital Universitario Virgen del Rocío/CSIC/ \\ Universidad de Sevilla, Sevilla, Spain
}

\begin{abstract}
Exposure of lung to hypoxia has been previously reported to be associated with significant alterations in the protein content of bronchoalveolar lavage (BAL) and lung tissue. In the present work we have used a proteomic approach to describe the changes in protein complement induced by moderate long-term hypoxia (rats exposed to $10 \% \mathrm{O}_{2}$ for 72 hours) in BAL and lung tissue, with a special focus on the proteins associated with pulmonary surfactant, which could indicate adaptation of this system to limited oxygen availability. The analysis of the general proteomic profile indicates a hypoxia-induced increase in proteins associated with inflammation both in lavage and lung tissue. Analysis at mRNA and protein levels revealed no significant changes induced by hypoxia on the content in surfactant proteins or their apparent oligomeric state. In contrast, we detected a hypoxia-induced significant increase in the expression and accumulation of hemoglobin in lung tissue, at both mRNA and protein levels, as well as an accumulation of hemoglobin both in BAL and associated with surface-active membranes of the pulmonary surfactant complex. Evaluation of pulmonary surfactant surface activity from hypoxic rats showed no alterations in its spreading ability, ruling out inhibition by increased levels of serum or inflammatory proteins.
\end{abstract}

\section{Introduction}

Provision of oxygen to the tissues, the main function of the lung, is achieved by gas exchange between air and blood, which occurs in the alveoli. Oxygen deficit leads to a severe impairment of tissue function, including alterations of the lung itself. The hypoxia

\footnotetext{
(C) 2014 Elsevier B.V. All rights reserved.

Corresponding author: Jesús Pérez-Gil, Dept. Bioquimica, Fac. Biologia, Universidad Complutense, José Antonio Novais 2 , 28040 Madrid, Spain, Ph.: +34 913944994, Fax: +34 913944672, jperezgil@bio.ucm.es.

Publisher's Disclaimer: This is a PDF file of an unedited manuscript that has been accepted for publication. As a service to our customers we are providing this early version of the manuscript. The manuscript will undergo copyediting, typesetting, and review of the resulting proof before it is published in its final citable form. Please note that during the production process errors may be discovered which could affect the content, and all legal disclaimers that apply to the journal pertain.
} 
response of the organism depends on severity and exposure time and includes two types of effects. Acute effects (seconds to minutes) are mediated through ion channel regulation, while chronic responses (hours to days), include several effects such as activation of glucose metabolism, erythropoiesis, angiogenesis, pulmonary hypertension (caused by vasoconstriction and vascular hypertrophy) and inflammation [1-4]. Chronic responses to hypoxia are mediated through induction of several transcription factors (hypoxia-inducible factors; HIFs), including the ubiquitously expressed HIF-1 and the tissue limited HIF-2 and HIF-3. HIF-1 binds to hypoxia responsive elements of several gene enhancers, such as vascular endothelial growth factor (VEGF), involved in vascular responses to hypoxia, and hypoxia induced mitogenic factor (HIMF), with angiogenic and vasoconstrictor effects [1, 5].

Several in vivo models of hypoxia have been previously described. Exposure of animals to $10 \%$ oxygen has been reported to induce several changes in the organism, including alteration of alveolar permeability [6]. Impairment of transalveolar fluid transport has been found to cause edema due to insufficient alveolar fluid clearance, though some authors reported that edema during the first hours decreases at longer exposure times [7]. In the same way, inflammation occurring because of reactive oxygen species (ROS) and albumin extravasation, could be resolved in a few days after exposure when vascular epithelium acclimates [8]. Referring to changes in protein expression induced by hypoxia, different temporal expression patterns have been found in animal models exposed to $10 \%$ oxygen, including increased expression of genes involved in immune responses and pulmonary vascular remodeling, occurring between days 1 and 7 of hypoxia exposure [9].

The main metabolically active lung epithelial cells are alveolar type II pneumocytes, which produce and secrete pulmonary surfactant. Interestingly, expression of $a$ and $\beta$ hemoglobin in type II cells has been reported, indicating potential functions of this protein in lung as an oxygen transporter, oxygen sensor or oxidative stress protector [10,11]. Recently, induction by hypoxia of hemoglobin expression in these cells has been demonstrated in vitro [12], suggesting the existence of an oxygen-sensing pathway in alveolar epithelial cells.

Pulmonary surfactant is a lipid-protein complex that lines the alveolar surface, and reduces surface tension at the air-fluid interface. This function is essential to stabilize the alveoli, prevent their collapse at the end of expiration, and avoid alveolar edema. Composition of surfactant includes about $90 \%$ lipids (mainly phospholipids) and 8-10\% of surfactantassociated proteins. Pulmonary surfactant is stored in type II alveolar epithelial cells in the form of densely packed bilayers called lamellar bodies that are secreted and efficiently transferred into the interface [13]. Lipid transport into lamellar bodies could be ultimately mediated by the transporter ABCA3. This protein seems to have an important role in lung and surfactant maturation $[14,15]$. The surface active function of interfacial surfactant films is mainly supported by its major phospholipid dipalmitoyl phosphatidylcholine (DPPC) (40-50\%) and the presence of hydrophobic surfactant proteins SP-B and SP-C [13, 16]. Hydrophilic surfactant proteins SP-A and SP-D participate in the innate immune response by binding to pathogens and activating alveolar macrophages. Besides its surface activity and defence function, it has been recently proposed that a proper structure of the pulmonary surfactant layer can also be important to optimize oxygen diffusion through the air-water 
respiratory interface [17]. It remains, therefore, an open question whether there is an optimization of surfactant to conditions of limited oxygen availability, and whether exposure to reduced oxygen partial pressures could affect composition and/or function of pulmonary surfactant.

In the present work we have performed a large-scale characterization of the overall proteomic pattern of lung tissue and bronchoalveolar lavage (BAL) from rats subjected to moderate long term hypoxia (10\% oxygen for 72 hours) using a two-dimensional difference gel electrophoresis (2D-DIGE) approach. We have also studied and compared mRNA expression of several hypoxia-related factors such as HIF-1a, HIMF, $a$-globin and $\beta$-globin, and surfactant-related proteins SP-A, SP-B, SP-C, SP-D, and ABCA3, in lung tissue from normoxic and hypoxic rats. Subsequently we compared the presence of these proteins in BAL from both groups of animals. Finally, to detect possible hypoxia-promoted changes in the surface activity of pulmonary surfactant we analyzed and compared the interfacial absorption capabilities of surfactant.

\section{Experimental Procedures}

\section{Sample collection}

Rats were maintained either under normoxic conditions $\left(21 \% \mathrm{O}_{2}\right)$ or in a hypoxia incubator for $72 \mathrm{~h}$ at $10 \% \mathrm{O}_{2}$. Animals were then anesthetized and perfused with saline, to remove circulating cells and serum proteins in order to avoid accidental blood contamination during the subsequent collection of BAL. After clamping the right mainstem bronchus the left lung was lavaged with $0.9 \% \mathrm{NaCl}$. The right lung was removed and cut into four pieces that were immediately frozen in liquid nitrogen. BAL was centrifuged at $150 \mathrm{~g}$ for 10 minutes at $4^{\circ} \mathrm{C}$ to remove cells and the supernatants were frozen.

To obtain whole native rat surfactant, BALs from both lungs were centrifuged for 1 hour at $100,000 \mathrm{~g}$ and membranes were cleaned to remove potential blood contaminants by centrifugation on a sodium bromide gradient, as previously described [18].

Alternatively, to separate the most surface active fraction of pulmonary surfactant, i.e. the large aggregates (LA), from the less active small aggregates (SA), BALs were centrifuged for 1 hour at 48,000 g, and the supernatants containing SA and pellets containing LA were kept frozen. Phospholipid content of the samples was determined by phosphorus assay, and total protein content was quantified by Lowry assay.

\section{RNA extraction}

Total RNA was isolated from frozen pulverized lung tissue by acid guanidine thiocyanate phenol-chloroform extraction with RNA-Bee reagent (Tel-Test, Inc.). Concentration of RNA was determined by absorbance at $260 \mathrm{~nm}$ measured in a NanoDrop spectrophotometer (Thermo Scientific).

\section{Real-time PCR}

After treatment with DNase I (Ambion) to destroy genomic DNA contamination, reverse transcription of RNA was performed with M-MLV Reverse Transcriptase (Invitrogen). PCR 
was performed under standard cycling conditions using reagents from Applied Biosystems. The ABI Prism 7900HT instrument and the Sequence Detection Software v2.2 (SDS 2.2) were used for PCR quantification and measurement of the PCR amplification product. Primers and probes used for the realtime PCR were obtained from Applied Biosystems (Taqman ${ }^{\circledR}$ Gene Assays) for 9 genes: Sftpa1 (Rn00824545_m1), Sftpb (Rn00593742_m1), Sftpc (Rn00569225_m1), Sftpd (Rn00563557_m1), Abca3 (Rn01461661_g1), Hif1a (Rn00577560_m1), Retnla (Rn00584229_m1), Gloa (Rn01463755_g1) and Hbb (Rn00583657_g1). The 18s rRNA (Hs99999901_s1) was used as endogenous control. Analysis and comparisons of the relative expression of genes coding for the different proteins selected were performed by the $2^{-\Delta \Delta C T}$ method [19].

\section{Electrophoresis and Western blot analysis}

SDS/PAGE was performed under reducing conditions, in the presence of $5 \% \beta$ mercaptoethanol. Samples loaded on each gel contained the same amount of phospholipid. For analysis of SP-B and SP-C, proteins separated by the gels were transferred to PVDF membranes (Bio-Rad Laboratories) using a wet transfer system at $300 \mathrm{~mA}$ for 1 hour. For the rest of the Western blots, proteins were transferred to nitrocellulose membranes (Amersham Biosciences) using a semi-dry transfer system at $20 \mathrm{~V}$ for 20 minutes. Blocking, washing and incubation with the antibodies were performed using a protein detection system under vacuum (SNAP i.d., Millipore Corp.). The primary antibodies used were: rabbit antiSP-B (1:5,000), rabbit anti-mature SP-C (1:7,000) (both from Seven Hills Bioreagents), rabbit anti-SP-A $(1: 2,000)$ (kindly supplied by Dr. J. Wright, from Duke University) and rabbit-anti hemoglobin $(1: 4,000)$ (Biogenesis). Secondary antibody was goat anti-rabbit HRP-conjugated $(1: 10,000)$ (Santa Cruz Biotechnology). Gels were analyzed using Quantity One software (Bio-Rad).

\section{Spreading assay}

Experiments were made in a specially designed custom-made NIMA surface balance with a trough capacity of $15 \mathrm{ml}$. Samples were carefully deposited onto the water phase with a Hamilton syringe and the increment of surface pressure was monitored over time by a Wilhelmy paper plate connected to a pressure sensor. The assays were carried out at $25^{\circ} \mathrm{C}$ using a subphase of $5 \mathrm{mM}$ Tris, $150 \mathrm{mM} \mathrm{NaCl}, \mathrm{pH} 7$.

\section{Protein sample preparation for 2D-DIGE}

Frozen lung tissue was pulverized and sonicated for 10 seconds in standard cell lysis buffer (30 mM Tris-HCl, 2M Thiourea, 7M Urea, 4\% CHAPS, $\mathrm{pH} 8.5$ ). The homogenates were centrifuged for 5 minutes at $150 \mathrm{~g}$ at $4^{\circ} \mathrm{C}$ and the protein concentration in the supernatants was determined using the Bio-Rad Micro Assay. BALs were concentrated by centrifugation in Agilent Spin Concentrators (Agilent Technologies), before being lyophilized and dissolved in cell lysis buffer. Protein content in the concentrated samples was determined using the Pierce Micro BCA Protein Assay. 


\section{Minimal labeling of proteins with CyDye DIGE Fluors for 2D-DIGE}

Two different experiments were performed, one with BAL ( 6 control and 6 hypoxic samples) and the other one with lung homogenate ( 6 control and 6 hypoxic samples). For each experiment, $50 \mu \mathrm{g}$ protein aliquots of each sample were labeled either with $\mathrm{Cy} 3$ or $\mathrm{Cy} 5$ following the manufacturer's protocol (GE Healthcare). A pool containing $750 \mu \mathrm{g}$ of protein was created by combining equal amounts of protein from every sample, and $350 \mu \mathrm{g}$ of this pool was labeled with Cy2. A $400 \mu \mathrm{g}$ aliquot from the protein pool was left unlabeled for the picking gel.

\section{Two-Dimensional Difference Gel Electrophoresis}

Information about the 2D-DIGE study is provided in a form (see Supplementary File 1) that complies with the most recent version of Minimum Information About a Proteomics Experiment - Gel Electrophoresis (MIAPE-GE) standards currently under development (<http://www.psidev.info/miape/MIAPE_GE_1_4.pdf>) by the Human Proteome Organization Proteomics Standards Initiative. For each gel, $50 \mu \mathrm{g}$ of a Cy3-labeled sample (from control or hypoxic animal), $50 \mu \mathrm{g}$ of a Cy5-labeled sample (from the other group of animals) and $50 \mu \mathrm{g}$ of the Cy2-labeled pool were mixed and an equal volume of $2 \mathrm{X}$ sample buffer containing 2M thiourea, $7 \mathrm{M}$ urea, $2 \% \mathrm{pH} 3-10 \mathrm{NL}$ IPG buffer, $4 \%$ CHAPS and $1.2 \%$ DeStreak Reagent (GE Healthcare) was added to all samples including the unlabeled pool. For picking gels, the final volume was brought up to $450 \mu \mathrm{l}$ with rehydration buffer (DeStreak Rehydration Solution (GE Healthcare), 0.5\% pH 3-10 NL IPG buffer). The first dimension of electrophoresis (isoelectric focusing) was done using an IPGphor II apparatus (GE Healthcare) on $24 \mathrm{~cm} \mathrm{pH} 3-10 \mathrm{NL}$ Immobiline DryStrips (GE Healthcare). For 2DDIGE experiments, samples were cup loaded after rehydrating the strips overnight for the analytical gels, whereas the samples were applied in the rehydration solution for the picking gels. After equilibration of the strips in equilibration solution I (50 mM Tris-HCl, $6 \mathrm{M}$ urea, $30 \%$ glycerol, $2 \%$ SDS, $0.5 \%$ dithiothreitol (DTT)) and equilibration solution II (containing $4.5 \%$ iodoacetamide instead of DTT) for 15 minutes each, second dimension separations were run on gradient 8-18\% polyacrylamide gels in Tris/glycine/SDS running buffer, using the Ettan DALTtwelve system (GE Healthcare). One of the plates of the picking gel sandwich was treated with Bind-Silane solution (80\% ethanol, $0.02 \%$ glacial acetic acid, $0.001 \%$ Bind-Silane) (GE Healthcare) to allow removal of the other plate after electrophoresis and staining of the gel with Deep Purple Total Protein Stain (GE Healthcare).

\section{Gel imaging and analysis}

Information about the acquisition and processing of data from the 2D-DIGE studies are provided in the form that complies with the most recent version of the guidelines established for Minimum Information about a Proteomics Experiment - Gel Informatics (MIAPE-GI) currently under development by the Human Proteome Organization Proteomics Standards Initiative http://www.psidev.info/files/miape-gi-v1.pdf (see Supplementary File 2). Gels were imaged using the Typhoon 9410 fluorescent imager (GE Healthcare). The images were analyzed with Progenesis SameSpots v2.0 (Nonlinear USA, Inc., Durham, NC) including alignment, spot detection and matching. Quantitation of normalized volumes for all spots 
was performed by comparing the ratio of each $\mathrm{Cy} 3$ or $\mathrm{Cy} 5$ value to the values obtained from the normalization pool/Cy2 channel present on each gel.

\section{Protein identification by mass spectrometry}

Protein spots were picked from the picking gel using the Ettan Spot Picker (GE Healthcare). The matching of individual spots from gel to gel was performed by the Progenesis SameSpots software. All matches were then reviewed and any necessary edits were made. The gel plugs were washed twice with $200 \mathrm{mM}$ ammonium bicarbonate, $40 \%$ acetonitrile for 30 minutes at $37^{\circ} \mathrm{C}$ and dehydrated with $75 \%$ acetonitrile for 20 minutes followed by air drying for 30 minutes at $30^{\circ} \mathrm{C}$. The proteins were then digested with trypsin (proteomics grade, Sigma) overnight at $37^{\circ} \mathrm{C}$. Fifty percent acetonitrile, $0.1 \%$ Trifluoroacetic Acid (TFA) was then added to the trypsin solution, incubated for 30 minutes at $37^{\circ} \mathrm{C}$, transferred to a new plate and dried by Speed Vac. The extracted peptides were re-suspended in $0.5 \%$ TFA and desalted and concentrated using C18 ZipTips (Millipore Corporation). The peptides were then spotted onto a 384-well MALDI plate and allowed to dry before Matrix Solution ( $2 \mathrm{mg} / \mathrm{ml}$ ACH Cinnamic Acid, $60 \%$ acetonitrile, $10 \mathrm{mM}$ ammonium phosphate monobasic) was added. Peptides were analyzed by MALDI-TOF/TOF mass spectrometry using a 4800 MALDI TOF/TOF Proteomics Analyzer (Applied Biosystems) in The Proteomics and Small Molecule Mass Spectrometry Core Facility at The Pennsylvania State University College of Medicine. MASCOT confidence interval scores of $>95 \%$ were considered a positive protein identification.

\section{Statistics}

For proteomics gel images, statistical analysis was performed using t-test and one way ANOVA analysis, and multivariate statistical analysis was done using the Progenesis Stats Module to perform Principal Components Analysis (PCA), Correlation Analysis, Clustering and Power Analysis of the data. For the rest of experiments statistical significance was evaluated by $t$-Student test. In all cases $p$-values $<0.05$ were considered statistically significant.

\section{Results}

\section{Validation of the hypoxia model}

To confirm that the hypoxia model used in this study induced a response in the rats, the relative mRNA expression of the hypoxia factors HIF-1a and HIMF in the lungs of control and hypoxic animals was determined by real-time PCR (Figure 1A). As expected, the levels of both mRNAs were significantly increased in the hypoxic animals.

Moreover, principal components analysis (PCA) performed with proteomic data (Figure 1B), which will be discussed later, clearly showed a separation of the groups (control and hypoxia) into distinct data regions. PCA was conducted using all the analyzed spots and also using only the significant spots in the two experiments carried out (BAL and tissue samples). Data from principal components 1 and 2 account for more than $70 \%$ of the variance for both comparisons. In the case of BAL samples (Figure 1B) even the analysis performed with all protein spots (left panel) revealed two different populations, as was 
observed when PCA was performed with only the significantly different spots (right panel). Therefore, PCA indicates that the selected hypoxic conditions actually induce a differential response in the animals that becomes apparent in the proteomic component.

\section{Effect of hypoxia on the lipid and protein contents of lung tissue and BAL}

As shown in Figure 2A, no significant changes were found in the total lipid content of samples from control and hypoxic rats. Nevertheless, the results indicate that hypoxic rats exhibit a trend towards higher phospholipid levels in pulmonary surfactant, both in LA (which correspond to the most active fraction of the material) and SA. The phospholipid (SA/LA) ratio between both fractions revealed no changes between the two groups of animals, an indication that hypoxic rats sustain an appropriate surfactant lipid metabolism. In addition, we did not find any changes in cholesterol content (not shown).

With regards to the protein content, Figure $2 \mathrm{~B}$ also illustrates that a significantly higher amount of total protein was detected in the BALs obtained from hypoxic rats. This was not accompanied, however, with significant changes in the content of protein associated to purified pulmonary surfactant, expressed either as total protein or as the protein to phospholipid ratio.

\section{Changes in mRNA and protein expression of lung proteins in response to hypoxia}

The relative mRNA expression of pulmonary surfactant proteins SP-A, SP-B, SP-C, SP-D and the lipid transporter ABCA3, involved in surfactant biogenesis, was determined by realtime PCR. The results showed that after 72 hours of hypoxia no changes in the mRNA levels of these surfactant-related proteins were observed (Figure 3A). Also, the relative amount of the main proteins associated with pulmonary surfactant complexes, SP-A, SP-B and SP-C, was studied by Western blot of samples from BAL from control and hypoxic animals. No significant differences were observed in the content of surfactant proteins, or in their apparent oligomerization state, between either group of animals (Figure 3B).

\section{Changes in mRNA expression and protein levels of hemoglobin in response to hypoxia}

The relative mRNA expression of $\alpha$ - and $\beta$-globin was determined by real-time PCR, showing that the lungs from hypoxic animals exhibited a significant increase in the mRNA levels coding for both hemoglobin subunits (Figure 4A). We also analyzed the relative amount of hemoglobin in BALs and pulmonary surfactant of samples from both control and hypoxic animals by Western blot. The results confirmed that animals exposed to hypoxia exhibit a significant increase in the hemoglobin content (in monomer and dimer forms), in both BAL and surfactant complexes, in comparison to the samples from normoxic rats (Figure 4B).

The proteomic study also revealed additional information about the relative presence of $a$ and $\beta$-globins. It is remarkable that in BAL, only the beta chain was found, with several of its isoforms with a molecular weight of $16 \mathrm{kDa}$ and different isoelectric points around 7.8 identified. Several spots of the $\beta$-globin with the same molecular weight as in BAL samples and isoelectric points between 6.7 and 7.8 were also found in tissue samples. In contrast to $\beta$ - chain, several isoforms of a-globin were only detected in tissue samples (Figure 5A). 
One spot had a molecular weight of approximately $27 \mathrm{kDa}$ and an isoelectric point of 8.4, and two more spots with approximate molecular weights of $17 \mathrm{kDa}$ and isoelectric points of 5 and 6 , respectively.

In BAL samples no statistical differences were found in the levels of p-globin when comparing control and hypoxia groups. In lung tissue, however, although the statistical analysis of total a-globin detected on the gels showed no significant differences in hypoxic rats, the independent analysis of the 3 spots identified as a-globin revealed significant changes (Figure 5B). A decrease in the levels of the $27 \mathrm{kDa}$ isoform (spot 4(c) in Figure 5A) was observed in hypoxic lungs, whereas the other two isoforms of a-chain were found increased in these animals.

\section{Effect of hypoxia on the interfacial function of pulmonary surfactant}

The increase observed in total protein content of BAL, including the higher amount of hemoglobin, both in lavage and surfactant, raised the question of a potential inactivation of surfactant, particularly with respect to interfacial adsorption, due to the association of serum and inflammatory proteins [18]. We therefore compared the ability of pulmonary surfactant from both animal groups to spread over and adsorb onto an air-liquid interface, by registering the kinetics of surface pressure increase with time. Figure 6 compares the interfacial adsorption of different amounts of spread surfactant. Surfactant from hypoxic rats produce higher surface pressures (lower surface tensions) than that from normoxic animals. Surfactant of animals exposed to hypoxia, purified from BALs containing higher protein-tolipid ratios, do not show inhibited, but rather slightly enhanced surface activity.

\section{Changes in the protein profile of BAL studied with 2D-DIGE}

Upon 2D-DIGE of samples from BAL of control and hypoxic rats, 657 protein spots were matched in both groups. By analyzing 249 of these spots (including all spots undergoing significant changes) by MALDI-ToF/ToF, we were able to identify 213 spots corresponding to a total of 41 proteins with MASCOT confidence interval scores of $>99 \%$ (see reference gel in Supplementary Figure 1 and Supplementary Table 1). It should be noted that in many cases there were multiple spots, usually representing different isoforms of the same protein. We have also included 2 proteins that had been previously identified by this laboratory in rat bronchoalveolar samples, and are shown with an asterisk in the table.

The sums of normalized volumes of all spots (isoforms) for each identified protein were then calculated. Analysis by t-test showed that the levels of 25 of these proteins (58\% of the proteins in the group) significantly $(\mathrm{p}<0.05)$ changed when comparing samples from hypoxic and normoxic animals, with 16 of them decreasing (37\%) and 9 increasing $(21 \%)$ in the hypoxic animals.

Percent changes of protein levels showing significant differences upon comparison of samples from normoxic and hypoxic animals are summarized in Table 1. Negative values indicate a reduction of the protein content in the hypoxic group with respect to the normoxic group. Particularly large are the changes revealed in the levels of ceruloplasmin and interalpha-inhibitor $\mathrm{H} 4$, both significantly increased in BAL from hypoxic animals. Other proteins also exhibited marked changes, such as abhydrolase, $\mathrm{Cu} / \mathrm{Zn}$ superoxide dismutase, 
glutathione S-transferase, haptoglobin, lipocortin I, NM23B, phosphatidylethanolamine binding protein and RAKc, all of them with a reduced level of more than $43 \%$ as a consequence of hypoxia.

\section{Changes in the protein profile of lung tissue}

A large number of protein spots $(\sim 1100)$ were matched on 2D-DIGE analysis of control and hypoxic groups for the lung homogenate samples (Sup. Figure 2 and Sup. Table 2). Of the 170 spots analyzed by MALDI-ToF/ToF, including all spots undergoing significant changes, 40 proteins were identified with confidence interval scores of $>95 \%$. Of these proteins, 16 were also identified in BAL samples. The t-test for the sums of normalized volumes revealed that only 7 proteins showed significant changes $(\mathrm{p}<0.05)$ in hypoxia $(17.5 \%$ of the total identified proteins resolved on the gels). Of them, 5 proteins had increased and 2 had decreased levels.

The percent changes of significantly changed proteins are summarized in Table 2 . In addition to the low number of significant changes found in the tissue homogenates when comparing the two animal groups, the percent changes are also relatively limited, with none of the 7 proteins changing more than $40 \%$.

\section{Categorization of proteins}

In order to analyze the general implications of the protein changes, we assigned all of the identified proteins to broad functional categories based on the published literature. The categories we formed included proteins involved in the immune response and its regulation (IMM), metabolism-related proteins (MET), cytoskeletal proteins (CYT), and proteins involved in redox balance (RED). It should be noted that some proteins were assigned to multiple functional groups. A graphic representation of these groups using Venn diagrams is given for BAL in Figure 7A for BAL and Figure 7B for lung tissue.

\section{Discussion}

A main motivation of the present study was to investigate whether the physiological adaptation to a reduced availability of oxygen in a mammalian lung could include an intrinsic alteration of the pulmonary surfactant system, at a compositional, structural or functional level. In a recent study, we had obtained evidence that the structure and protein composition of pulmonary surfactant membranes could have some impact on the oxygen diffusion properties of the air-water interface [17]. We expected that, if this is the case, adaptations to hypoxia could perhaps include alterations of the protein complement associated with surfactant. For this reason we chose a model of moderate long-term hypoxia conditions and a time point that allowed for the incorporation of long-term responses involving protein expression as promoted by well-defined hypoxia factors. In our model of rats exposed to $10 \%$ oxygen during 72 hours, we clearly detected an increase in mRNA expression of hypoxia inducible factors HIF-1a and HIMF, as found in other similar hypoxia [20, 21]. 


\section{Effect of hypoxia on the proteomic profile of BAL and lung tissue}

A result of the present study is that a markedly higher number of proteins changed significantly in BAL from hypoxic versus normoxic rats ( 25 proteins) compared to the differences in tissue homogenates (7 proteins). This particularly high plasticity of BAL proteome could reflect the confluence at the alveolar spaces of different hypoxia-affected processes, all of them somehow contributing to a large number of changes in protein complement. Filtration of proteins from plasma to the airways because of vascular remodelling, secretion of proteins by the epithelium or leakage of proteins due to disruption of the integrity of pneumocytes or macrophages, could all contribute to generate a differential hypoxia-induced alveolar proteome, which should be particularly patent at the BAL. In fact, comparison of proteins altered in BAL and tissue shows that some of the proteins clearly altered in BAL exhibit unaltered levels in lung tissue: albumin, gelsolin, lipocortin I, peroxiredoxin 6, protein RAKc and selenium binding protein 1 .

In order to analyze the main effects of hypoxia on the composition of BAL and tissue, the proteins exhibiting significant changes have been classified, based on the literature, into four functional groups (see Sup. Table 3). Most of the proteins whose levels showed significant hypoxia-induced changes can be assigned to more than one of these groups because of their multiple functions, as it is illustrated in Figure 7. For instance, it's worth mentioning the presence of haptoglobin (protein \#20) in the center of the diagram in Figure 9, exhibiting more than 1.5 fold reduction in the BALs of hypoxic rats. Indeed, haptoglobin is expressed and secreted by type II pneumocytes and alveolar macrophages, and from its co-localization with SP-B in lamellar bodies this protein has been recently suggested to be part of the surfactant system [22]. Haptoglobin has an anti-inflammatory, antioxidant, detoxifying and angiogenic role, which is usually increased in BAL as a consequence of lung injury [23]. Perhaps haptoglobin is a main actor at the pro-inflammatory response in a high-oxygen context but not so much under hypoxia.

Besides haptoglobin, some of the proteins we found at increased or decreased levels in BAL or lung tissue from hypoxic rats have been previously reported to be somehow related to surfactant. Calreticulin is expressed in type II cells and bind surfactant proteins SP-A and SP-D to modulate inflammation [24]. Its increase after 72 hours of hypoxia could be therefore considered as a marker of lung inflammation. Peroxiredoxin 6 is a peroxidase with phospholipase A2 activity, which is responsible for degradation of DPPC in type II pneumocytes and is inhibited by SP-A [25, 26]. The reduced levels of peroxiredoxin 6 in BAL samples from hypoxic rats could suggest a reduced metabolic rate of pulmonary surfactant inside the cell. Finally, lipocortin or annexin I is also synthesized by type II cells and alveolar macrophages and has an anti-inflammatory role due to its inhibitory action on phospholipase A2 [27]. Reduced levels of annexin I in BAL from hypoxic rats could therefore be associated with alterations in cellular integrity of type II pneumocytes or reveal a pro-inflammatory response in the lung.

Some of the proteins with levels affected had been previously reported as being altered during different models of hypoxia, as it occurs, for instance, with the protein exhibiting the highest percent of change (134.7\%) in our hypoxic rats, ceruloplasmin [28, 29], or others such as $\mathrm{Cu} / \mathrm{Zn}$ superoxide dismutase [30], calreticulin [31] or grp75, a heat shock protein 
that has been reported to be increased in an in vitro hypoxia model [32]. Some of the proteins altered in our study have a clear connection with inflammatory events occurring under pathological situations (proteins in group IMM). We have found some increase in response to hypoxia of the levels of anti-inflammatory proteins such as alpha-1-inhibitor III precursor [33], serine protease inhibitors 1 and $2 b$ [34], inter-alpha-inhibitor, calreticulin and grp75. In the MET group we found reduced levels of some proteins involved in lipid metabolism such as annexins A1 and A5, Pcyt 2 (phosphoethanolamine cytidyltransferase), PEBP (phosphatidylethanolamine binding protein) and peroxiredoxin 6 , and proteins involved in the metabolism of other molecules such as the protein expressed in nonmetastatic cells 2 (nucleoside diphosphate kinase B) and the tyrosine 3-monooxygenase/ tryptophan 5-monooxygenase activator protein (involved in serotonin and catecholamine synthesis). Down-regulation of metabolism is a known effect of hypoxia, with the aim of saving energy in a situation where ATP cannot be obtained by oxidative pathways. Cytoskeletal proteins (group CYT) of the endothelial cells are known to regulate pulmonary vascular permeability that is severely affected by hypoxia. Therefore the presence of some of these proteins in BAL is not unexpected as they are potentially released due to some kind of cell damage. Finally, we have found alterations in the levels of proteins related to the REDOX balance and detoxification processes (group RED). These changes include proteins with a detoxification function because of their ability to bind iron, a role particularly important due to the increase of the hematopoiesis induced by exposure to hypoxia [30]: albumin, ceruloplasmin, hemopexin and transferrin. Some antioxidant proteins were found at increased levels in the samples from hypoxic rats, such as calreticulin and peroxiredoxin 4. Other proteins, also with antioxidant functions, are however downregulated, such as $\mathrm{Cu} / \mathrm{Zn}$ superoxide dismutase, glutathione-s-transferase [34] and peroxiredoxin 6.

In summary, the overall proteomic analysis indicates that exposure to hypoxia for 72 hours induces a response in the lung mainly characterized by tissue inflammation, leakage of plasma proteins to the air spaces due to the alteration of vascular cell cytoskeleton, changes in REDOX balance by alteration of antioxidant proteins levels, and decrease of the metabolic processes. The liberation of serum and inflammatory proteins into the airways could then impose a major challenge to breathing mechanics, particularly if there is a severe impact on pulmonary surfactant performance.

\section{Effect of hypoxia on pulmonary surfactant}

Alteration of surfactant subtypes has been correlated in the literature with lung injury, as in case of ARDS [35]. In our study, the phospholipid ratio SA/LA remains unchanged after 72 hours of hypoxia, revealing that pulmonary surfactant turnover is not severely altered as a consequence of extensive inflammation, lung injury and/or perturbation of the alveolar capillary barrier. Moreover, although not significant, we detected a trend towards a higher phospholipid content of total pulmonary surfactant in animals exposed to hypoxia, suggesting that production and accumulation of higher amounts of surfactant could be part of the adaptive response. This hypothesis could be reasonable considering the role of hypoxia (at least in a perinatal context) in the regulation of alveolar maturation and phospholipid production by type II pneumocytes [36]. 
In our model, the mRNA levels of surfactant proteins SP-A, SP-B, SP-C, and SP-D as well as that of the lipid transporter $\mathrm{ABCA} 3$ remained unchanged after 72 hours of hypoxia. Likewise, we did not detect changes either in the relative amounts of the main proteins associated with surfactant complexes, SP-A, SP-B and SP-C, in BAL, as evaluated by Western blot. We did not determined whether the conditions of hipoxia tested could alter the processing and the levels of intermediates of surfactant proteins along their maturation pathway. Although we do not think this is probable, considering the invariable levels found of both the mRNA expression and the amounts of secreted surfactant proteins in the lavage, it remains to be analyzed in future studies whether prolonged hypoxia and concomitant inflammation could end in cellular stress and marked impairment of protein synthesis and processing. Still, our data suggest a limited effect of hypoxia on the structure and composition of the surfactant system itself, particularly at the level of its protein moiety and for the hypoxia conditions tested here. It remains to be seen whether longer exposures to hypoxia could induce larger effects.

Although the intrinsic protein composition of surfactant does not seem to be very much altered upon exposure to the hypoxia level assessed here, we could detect a significant increase in the total protein content in BAL from hypoxic rats, which could be a consequence of the inflammatory response in the lung causing alteration of vascular epithelium and leakage of serum proteins to the airways [37]. This increase in protein content is not accompanied by a significant increase in the total protein or the protein-tolipid ratio of pulmonary surfactant complexes themselves, reflecting that the main part of the proteins appearing in hypoxic lavages is not necessarily associated with pulmonary surfactant complexes. We have shown that surfactant of our hypoxic animals is not impaired but actually exhibits enhanced capacities to adsorb rapidly to the interface. It remains to be determined whether this apparently enhanced performance could be associated with adaptive changes in the molecular lipid composition, in the lateral structure or in the threedimensional organization of pulmonary surfactant membranes and complexes, with no profound changes in the surfactant protein levels.

\section{Effect of hypoxia on hemoglobin accumulation at the airways}

In spite of very slight alterations induced by hypoxia on the protein composition of pulmonary surfactant, we have detected a consistent and significant increase in the amount of hemoglobin associated with pulmonary surfactant complexes. This hemoglobin is strongly associated with surfactant membranes, because it remains as part of surfactant complexes after the differential centrifugation steps required to purify surfactant, including a gradient density centrifugation that permits purification of membranes with the particular low density (and low protein-to-lipid ratio) of surfactant, separately from any other cell membrane. This surfactant-associated hemoglobin does not seem to have any deleterious effect on pulmonary surfactant function, at least with respect to its ability to form high surface-active interfacial films.

Association of hemoglobin with surfactant complexes has been observed before, but has always been interpreted as a consequence of the eventual contamination of surfactant with blood, either because of lung edema or due to operational procedures during the collection 
of BAL and surfactant purification. We have taken all precautions to minimize blood contamination of BAL, including carrying out extensive perfusion of animals with saline prior to BAL and lung removal. The lavages obtained were judged to be practically free of blood contamination, and surfactant was still purified through steps that ensured removal of any other non-surfactant structures. We propose that at least part of the hemoglobin found associated with pulmonary surfactant might become part of surfactant membranes early during or after their synthesis by alveolar type II cells, which have the ability to express and produce hemoglobin, as it has been previously reported $[10,11]$. This is supported by the existence of hemoglobin also in the pulmonary surfactant from control rats, which should not in principle show alteration of vascular permeability, and where plasma extravasation should not be a source of the protein. Grek et al. reported induction of hemoglobin expression in type II cells upon exposure to hypoxia in vitro [12]. The increase of hemoglobin we have found in BAL-and surfactant, accompanied by an increase in the mRNA expression of both $\alpha$ - and $\beta$-globin in the lungs from our rats exposed to hypoxia, suggest that hemoglobin levels in lung could in fact be part of a regulated response to hypoxia, extending the existence of this hemoglobin-related response to the whole tissue and in an in vivo context.

On the other hand, our proteomic analysis reveals that the globins associated with pulmonary surfactant might differ from those constituting circulating blood hemoglobin. We were able to detect hemoglobin beta chain both in BAL and tissue, but we were not able to detect $a$-globin in the BAL samples. This fact could suggest that both $a$ and $\beta$ chains are expressed in lung tissue but that only $\beta$-globin might be finally secreted in BAL, and possibly integrated into the pulmonary surfactant complexes. Furthermore, the statistical analysis of the volume occupied by the spots identified as a-globin in the gels from lung tissue, revealed significant differences in the properties of this chain upon comparison of samples from normoxic and hypoxic rats. A higher molecular mass $(27 \mathrm{kDa})$ and $\mathrm{pI}(8.4)$ spot could correspond to a dimer of a-globins, as the molecular mass of the alpha chain is $15 \mathrm{kDa}$. Recently Ishikawa et al. found a-globin complexes in human lung tissue by 2D electrophoresis [38]. These complexes were reduced in lungs from idiopathic pulmonary fibrosis patients and prevention of complex formation was suggested to be due to a modified thiol group as a consequence of oxidative/nitrosidative stress. Our finding of a reduced level of the $27 \mathrm{kDa}$ a-globin complex in hypoxic lung could therefore be related to a potential oxidative stress caused by hypoxia. An extensive examination of the differential presence of $\mathbf{a}$ and $\beta$ globin in lung tissue and surfactant complexes, in response to particular physiological conditions, is hampered by the lack of inter-species specific antibodies for each of the globin forms. Future experiments will design alternative strategies to assess this question in detail.

Finally, it is important to mention that although vertebrate hemoglobins are heterotetramers of two alpha and 2 beta chain subunits, many species are able to synthesize different isoforms of hemoglobin, in such a way that adjustments in the stoichiometry of the different complexes may have effects on oxygen transport under certain hypoxic conditions [39]. In this respect, the stoichiometry of hemoglobin complexes could also be different in non- 
hematopoietic tissues [40] responding to concrete functional adaptations such as oxygen transport or sensing in the lung.

In summary, the present study raises the question of a potential specialized role of hemoglobin in respiratory physiology, which could be mediated upon assembly of certain globin forms as part of the pulmonary surfactant complex. Pneumocytes could thus be in charge of integrating environmental signals to modulate production and assembly of hemoglobin-loaded pulmonary surfactant, for which the ability to simultaneously optimize breathing mechanics and oxygen diffusion capabilities has still to be fully evaluated. The limited availability of material has prevented the comparison of oxygen diffusion properties of the surfactant from normoxic and hypoxic animals. Future experiments could assess whether supplementation of surfactant with hemoglobin has any impact on surface activity and oxygen diffusion properties of well defined pulmonary surfactant preparations.

\section{Supplementary Material}

Refer to Web version on PubMed Central for supplementary material.

\section{Acknowledgments}

This research has been supported by grants from the Spanish Ministry of Science (BIO2012-30733, CSD2007-00010), the Regional Government of Madrid (S2009MAT-1507) and NIH HL34788.

\section{References}

1. Lopez-Barneo J, Pardal R, Ortega-Saenz P. Cellular mechanism of oxygen sensing. Annu Rev Physiol. 2001; 63:259-87. [PubMed: 11181957]

2. Weir EK, Lopez-Barneo J, Buckler KJ, Archer SL. Acute oxygen-sensing mechanisms. N Engl J Med. 2005; 353:2042-55. [PubMed: 16282179]

3. Jain M, Sznajder JI. Effects of hypoxia on the alveolar epithelium. Proc Am Thorac Soc. 2005; 2:202-5. [PubMed: 16222038]

4. Bhattacharya J, Matthay MA. Regulation and repair of the alveolar-capillary barrier in acute lung injury. Annu Rev Physiol. 2013; 75:593-615. [PubMed: 23398155]

5. Semenza GL. Pulmonary vascular responses to chronic hypoxia mediated by hypoxia-inducible factor 1. Proc Am Thorac Soc. 2005; 2:68-70. [PubMed: 16113471]

6. Suzuki S, Noda M, Sugita M, Ono S, Koike K, Fujimura S. Impairment of transalveolar fluid transport and lung $\mathrm{Na}(+)-\mathrm{K}(+)-A T P a s e$ function by hypoxia in rats. J Appl Physiol. 1999; 87:962-8. [PubMed: 10484564]

7. Rassler B, Marx G, Reissig C, Rohling MA, Tannapfel A, Wenger RH, et al. Time course of hypoxia-induced lung injury in rats. Respir Physiol Neurobiol. 2007; 159:45-54. [PubMed: 17597012]

8. Gonzalez NC, Wood JG. Alveolar hypoxia-induced systemic inflammation: what low PO(2) does and does not do. Adv Exp Med Biol. 2010; 662:27-32. [PubMed: 20204767]

9. Wu W, Dave NB, Yu G, Strollo PJ, Kovkarova-Naumovski E, Ryter SW, et al. Network analysis of temporal effects of intermittent and sustained hypoxia on rat lungs. Physiol Genomics. 2008; 36:2434. [PubMed: 18826996]

10. Bhaskaran M, Chen H, Chen Z, Liu L. Hemoglobin is expressed in alveolar epithelial type II cells. Biochem Biophys Res Commun. 2005; 333:1348-52. [PubMed: 15979582]

11. Newton DA, Rao KM, Dluhy RA, Baatz JE. Hemoglobin is expressed by alveolar epithelial cells. J Biol Chem. 2006; 281:5668-76. [PubMed: 16407281] 
12. Grek CL, Newton DA, Spyropoulos DD, Baatz JE. Hypoxia Upregulates Expression of Hemoglobin in Alveolar Epithelial Cells. Am J Respir Cell Mol Biol. 2010

13. Perez-Gil J, Keough KM. Interfacial properties of surfactant proteins. Biochim Biophys Acta. 1998; 1408:203-17. [PubMed: 9813327]

14. Ban N, Matsumura Y, Sakai H, Takanezawa Y, Sasaki M, Arai H, et al. ABCA3 as a lipid transporter in pulmonary surfactant biogenesis. J Biol Chem. 2007; 282:9628-34. [PubMed: 17267394]

15. Stahlman MT, Besnard V, Wert SE, Weaver TE, Dingle S, Xu Y, et al. Expression of ABCA3 in developing lung and other tissues. J Histochem Cytochem. 2007; 55:71-83. [PubMed: 16982851]

16. Perez-Gil J, Weaver TE. Pulmonary surfactant pathophysiology: current models and open questions. Physiology (Bethesda). 2010; 25:132-41. [PubMed: 20551227]

17. Olmeda B, Villen L, Cruz A, Orellana G, Perez-Gil J. Pulmonary surfactant layers accelerate O(2) diffusion through the air-water interface. Biochim Biophys Acta. 2010; 1798:1281-4. [PubMed: 20227386]

18. Taeusch HW, Bernardino de la Serna J, Perez-Gil J, Alonso C, Zasadzinski JA. Inactivation of pulmonary surfactant due to serum-inhibited adsorption and reversal by hydrophilic polymers: experimental. Biophys J. 2005; 89:1769-79. [PubMed: 15923228]

19. Livak KJ, Schmittgen TD. Analysis of relative gene expression data using real-time quantitative PCR and the 2(-Delta Delta C(T)) Method. Methods. 2001; 25:402-8. [PubMed: 11846609]

20. Palmer LA, Semenza GL, Stoler MH, Johns RA. Hypoxia induces type II NOS gene expression in pulmonary artery endothelial cells via HIF-1. Am J Physiol. 1998; 274:L212-9. [PubMed: 9486205]

21. Teng X, Li D, Champion HC, Johns RA. FIZZ1/RELMalpha, a novel hypoxia-induced mitogenic factor in lung with vasoconstrictive and angiogenic properties. Circ Res. 2003; 92:1065-7. [PubMed: 12714564]

22. Abdullah M, Goldmann T. Pulmonary haptoglobin ( $\mathrm{pHp}$ ) is part of the surfactant system in the human lung. Diagn Pathol. 2012; 7:158. [PubMed: 23164167]

23. Zhang L, Wang M, Kang X, Boontheung P, Li N, Nel AE, et al. Oxidative stress and asthma: proteome analysis of chitinase-like proteins and FIZZ1 in lung tissue and bronchoalveolar lavage fluid. J Proteome Res. 2009; 8:1631-8. [PubMed: 19714806]

24. Kuroki Y, Takahashi M, Nishitani C. Pulmonary collectins in innate immunity of the lung. Cell Microbiol. 2007; 9:1871-9. [PubMed: 17490408]

25. Wu YZ, Manevich Y, Baldwin JL, Dodia C, Yu K, Feinstein SI, et al. Interaction of surfactant protein A with peroxiredoxin 6 regulates phospholipase A2 activity. J Biol Chem. 2006; 281:7515-25. [PubMed: 16330552]

26. Fisher AB, Dodia C. Lysosomal-type PLA2 and turnover of alveolar DPPC. Am J Physiol Lung Cell Mol Physiol. 2001; 280:L748-54. [PubMed: 11238016]

27. Gerke V, Moss SE. Annexins: from structure to function. Physiol Rev. 2002; 82:331-71. [PubMed: 11917092]

28. Martin F, Linden T, Katschinski DM, Oehme F, Flamme I, Mukhopadhyay CK, et al. Copperdependent activation of hypoxia-inducible factor (HIF)-1: implications for ceruloplasmin regulation. Blood. 2005; 105:4613-9. [PubMed: 15741220]

29. Healy J, Tipton K. Ceruloplasmin and what it might do. J Neural Transm. 2007; 114:777-81. [PubMed: 17406962]

30. White C, Kambe T, Fulcher YG, Sachdev SW, Bush AI, Fritsche K, et al. Copper transport into the secretory pathway is regulated by oxygen in macrophages. J Cell Sci. 2009; 122:1315-21. [PubMed: 19351718]

31. Jia L, Xu M, Zhen W, Shen X, Zhu Y, Wang W, et al. Novel anti-oxidative role of calreticulin in protecting A549 human type II alveolar epithelial cells against hypoxic injury. Am J Physiol Cell Physiol. 2008; 294:C47-55. [PubMed: 17959730]

32. Flores-Diaz M, Higuita JC, Florin I, Okada T, Pollesello P, Bergman T, et al. A cellular UDPglucose deficiency causes overexpression of glucose/oxygen-regulated proteins independent of the endoplasmic reticulum stress elements. J Biol Chem. 2004; 279:21724-31. [PubMed: 15020602] 
33. Greene CM, Miller SD, Carroll T, McLean C, O'Mahony M, Lawless MW, et al. Alpha-1 antitrypsin deficiency: a conformational disease associated with lung and liver manifestations. $\mathrm{J}$ Inherit Metab Dis. 2008; 31:21-34. [PubMed: 18193338]

34. Pastor MD, Nogal A, Molina-Pinelo S, Melendez R, Romero-Romero B, Mediano MD, et al. Identification of oxidative stress related proteins as biomarkers for lung cancer and chronic obstructive pulmonary disease in bronchoalveolar lavage. Int J Mol Sci. 2013; 14:3440-55. [PubMed: 23389041]

35. Veldhuizen RA, McCaig LA, Akino T, Lewis JF. Pulmonary surfactant subfractions in patients with the acute respiratory distress syndrome. Am J Respir Crit Care Med. 1995; 152:1867-71. [PubMed: 8520748]

36. Huang Y, Kempen MB, Munck AB, Swagemakers S, Driegen S, Mahavadi P, et al. Hypoxiainducible factor 2alpha plays a critical role in the formation of alveoli and surfactant. Am J Respir Cell Mol Biol. 2012; 46:224-32. [PubMed: 22298531]

37. Meyer KC, Zimmerman JJ. Inflammation and surfactant. Paediatr Respir Rev. 2002; 3:308-14. [PubMed: 12457601]

38. Ishikawa N, Ohlmeier S, Salmenkivi K, Myllarniemi M, Rahman I, Mazur W, et al. Hemoglobin alpha and beta are ubiquitous in the human lung, decline in idiopathic pulmonary fibrosis but not in COPD. Respir Res. 2010; 11:123. [PubMed: 20836851]

39. Storz JF, Moriyama H. Mechanisms of hemoglobin adaptation to high altitude hypoxia. High Alt Med Biol. 2008; 9:148-57. [PubMed: 18578646]

40. Mansergh FC, Hunter SM, Geatrell JC, Jarrin M, Powell K, Evans MJ, et al. Developmentally regulated expression of hemoglobin subunits in avascular tissues. Int J Dev Biol. 2008; 52:873-86. [PubMed: 18956317] 


\section{Significance}

This work reveals that hypoxia induces extensive changes in the proteomic profile of lung bronchoalveolar lavage, including the presence of proteins related with inflammation both in lung tissue and lavage, and a significant increase in the synthesis and secretion by the lung tissue of different forms of hemoglobin. The level of specific pulmonary surfactant-associated proteins is not substantially altered due to hypoxia, but hypoxia-adapted surfactant exhibits an enhanced ability to form surface-active films at the air-liquid interface. The increased amount of $\beta$-globin integrated into the operative surfactant complexes obtained from hypoxic rats is a relevant feature that points to the existence of adaptive responses coupling surfactant function and oxygen availability. 


\section{Highlights}

- Effect of hypoxia $\left(72 \mathrm{~h} 10 \% \mathrm{O}_{2}\right)$ has been analyzed in lung tissue and BAL proteome

- Hypoxia increased proteins associated with inflammation in BAL and lung tissue

- Hypoxia did not alter levels of surfactant-associated proteins

- Hypoxia promoted production of lung haemoglobin and its incorporation in surfactant

- Hypoxic surfactant showed $\geq$ interfacial adsorption rate than normoxic surfactant 
A. mRNA expression of hypoxia inducible factors in lung tissue
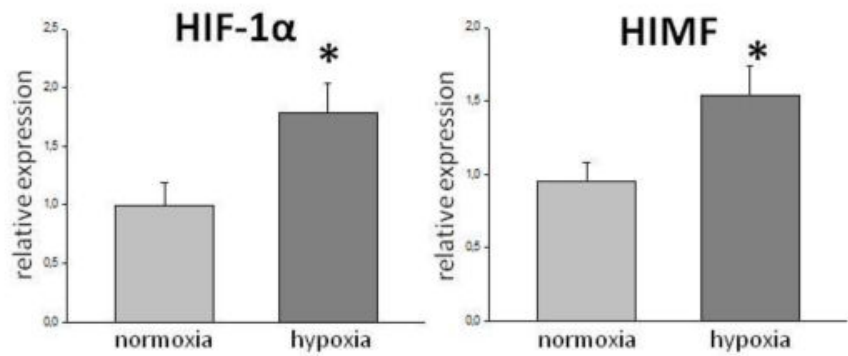

\section{B. Principal component analysis of proteomic data from BAL}
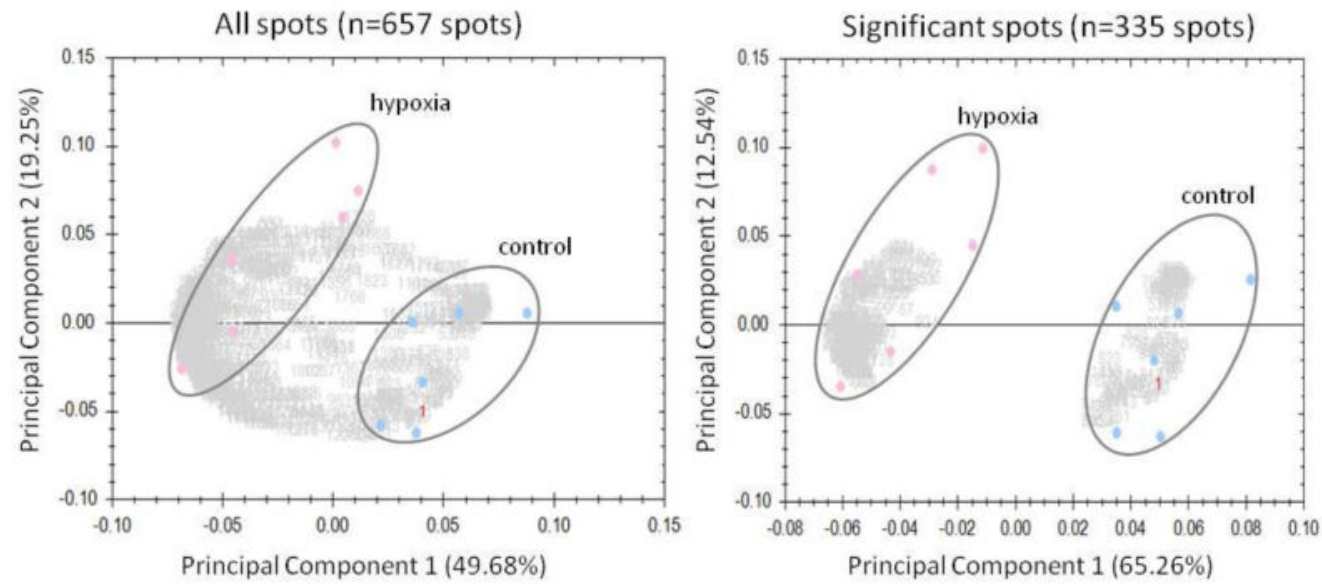

Figure 1. Validation of a moderate long-term hypoxia model (10\% oxygen exposure for 72 hours)

A. Induced mRNA expression of HIF-1a and HIMF hypoxia factors in lung tissue upon hypoxia exposure, as determined by real-time PCR. B. Principal component analysis of proteomic data revealed a clear separation between control and hypoxia groups. Dots represent the 6 animals studied in each group. 


\section{A. Lipid determinations}
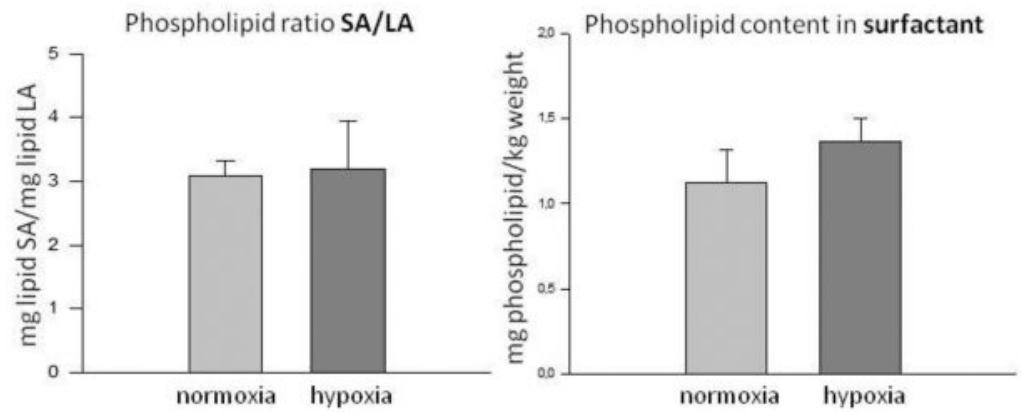

\section{B. Protein determinations}
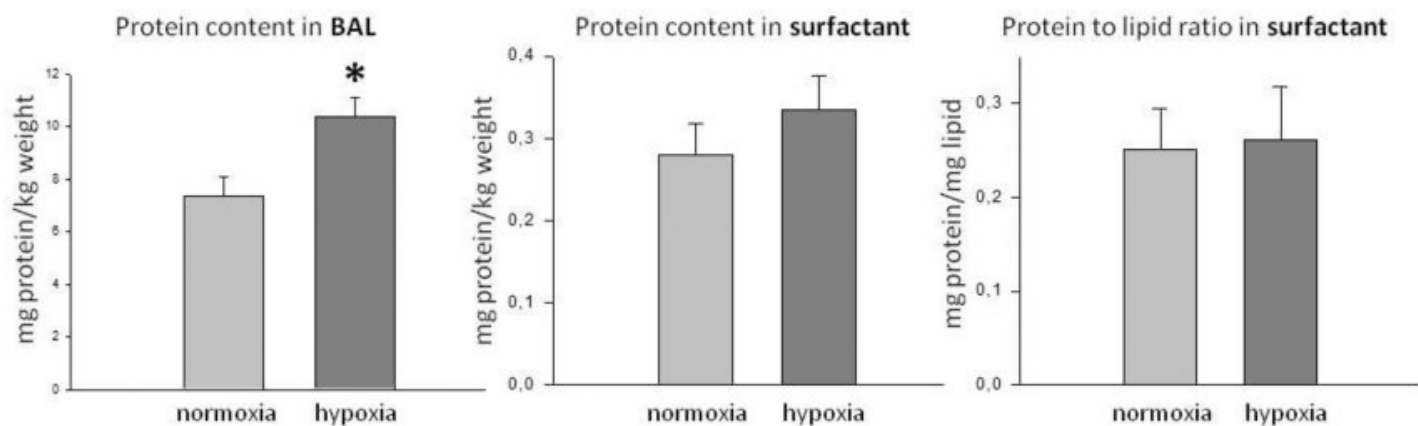

Figure 2. Effect of hypoxia in phospholipid and protein content of surfactant and BAL

$\boldsymbol{A}$. Balance of phospholipids in the small (SA) with respect to the large (LA) surfactant aggregates and total lipid content in pulmonary surfactant from control and hypoxic rats. $\boldsymbol{B}$. Total protein content in bronchoalveolar lavage (BAL) and purified surfactant, as well as the relative protein to lipid ratio in surfactant, as determined in BAL and surfactant from normoxic and hypoxic rats. Values are presented as means with standard error of the mean (SEM) ( $\mathrm{n}=6$ for each group), and significant differences are shown as $*(p<0.05)$. 
A. Changes in mRNA expression in lung tissue
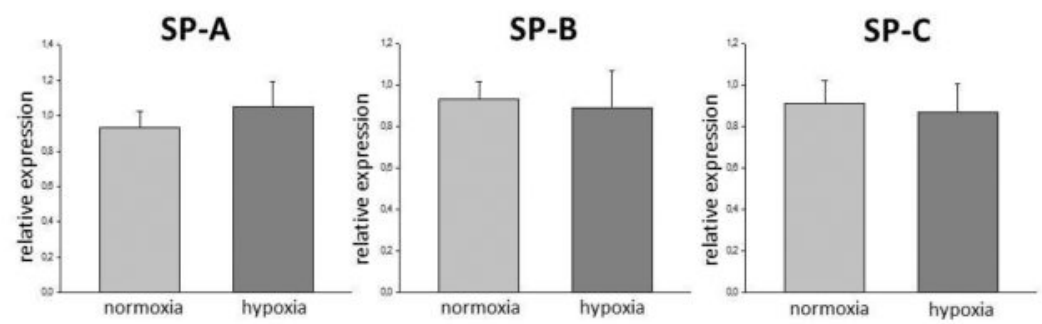

SP-D
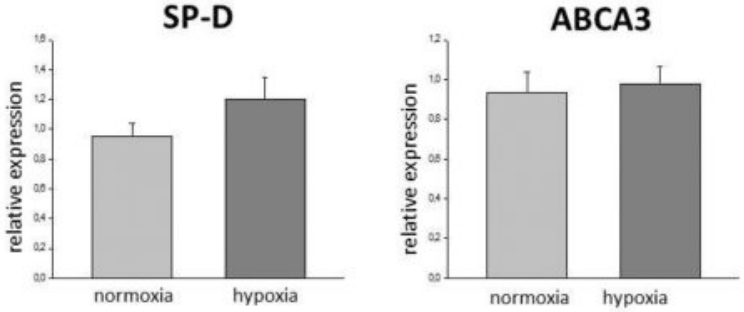

B. Changes in surfactant proteins content in BAL
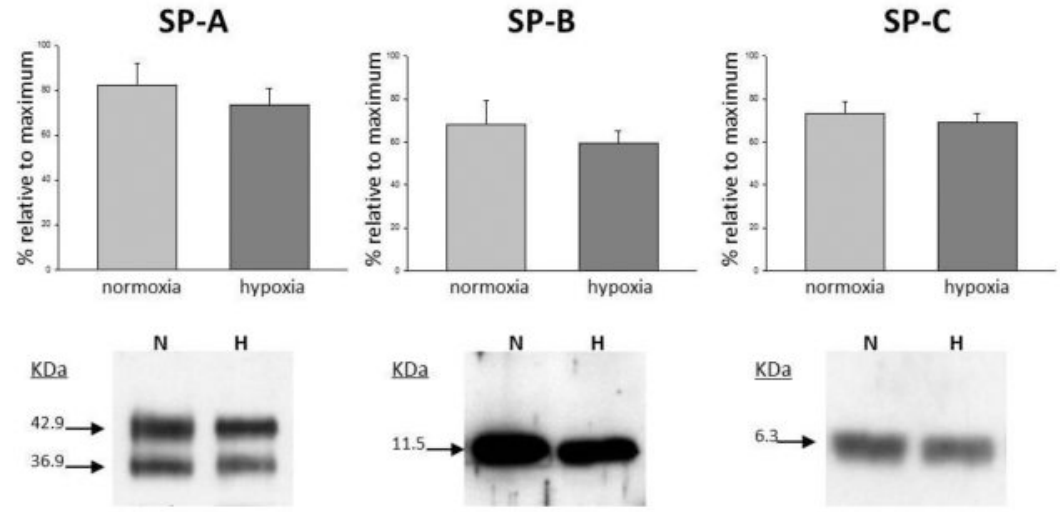

Figure 3. Effect of hypoxia on the mRNA expression and protein levels of surfactant-related proteins

A. Comparison of the relative expression of the genes coding for pulmonary surfactant proteins $\mathrm{A}, \mathrm{B}, \mathrm{C}$ and $\mathrm{D}$ and the lipid transporter $\mathrm{ABCA} 3$ involved in surfactant biogenesis, in control and hypoxic lung tissue, as determined by real-time PCR. Values are presented as means with standard error of the mean (SEM) ( $\mathrm{n}=6$ for each group). B. Comparison of the relative content of the main surfactant apolipoproteins as determined by Western blot of samples from bronchoalveolar lavage (BAL). Values are presented as means with SEM ( $n=4$ gels quantified by densitometry, with 6 rats from each group per gel). 
A. Changes in globins mRNA expression in lung tissue
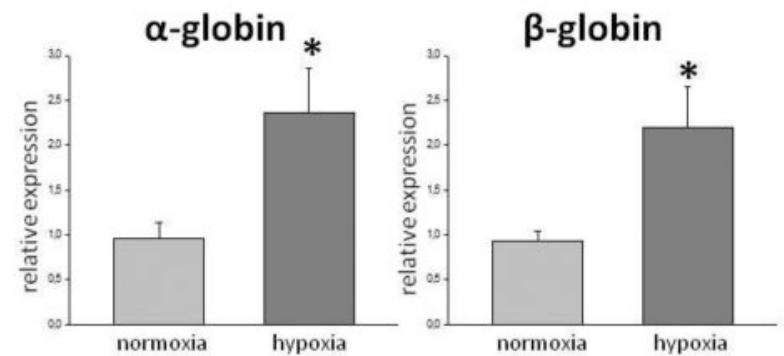

B. Changes in hemoglobin content in BAL and pulmonary surfactant
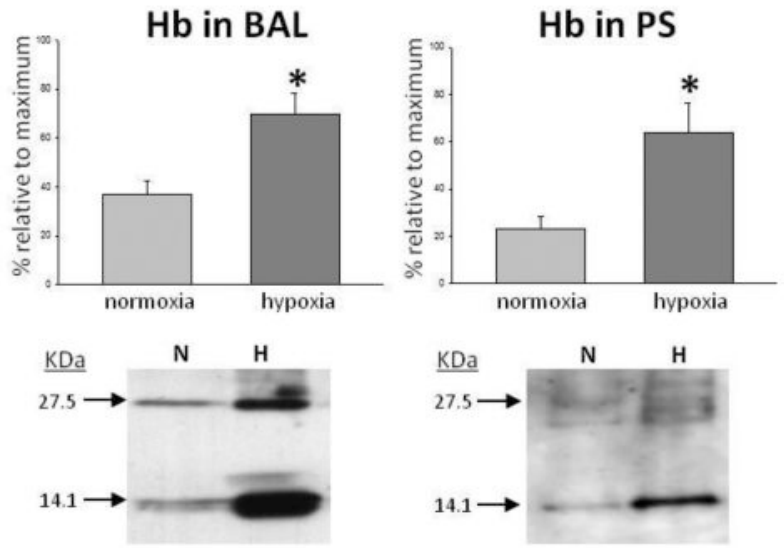

Figure 4. Changes in mRNA and protein levels of hemoglobin in response to hypoxia

A. Comparison of the relative expression of globin genes in control and hypoxic lung tissue, as determined by real-time PCR. Values are presented as means with standard error of the mean (SEM) ( $\mathrm{n}=6$ for each group) and significant differences are shown as $*(p<0.05)$. B.

Comparison of the relative content of hemoglobin as determined by Western blot of samples from bronchoalveolar lavage (BAL) and pulmonary surfactant (PS). Values are presented as means with SEM ( $\mathrm{n}=4$ gels quantified by densitometry of $14 \mathrm{kDa}$ bands, with 6 rats from each group per gel). Significant differences are shown as $*(p<0.05)$. 


\section{A. Alpha-globin spots in tissue}

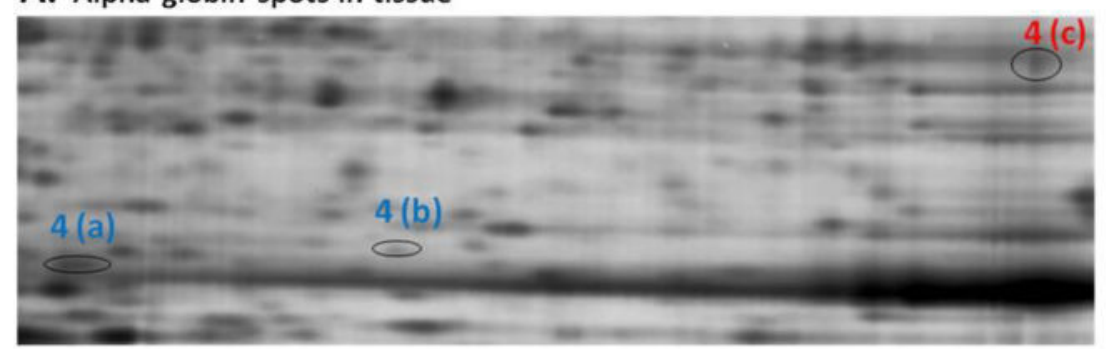

B. Normalized volumes of alpha-globin spots in tissue

\begin{tabular}{|c|c|c|c|}
\hline Spot & $\begin{array}{c}\text { Control } \\
(\text { mean } \pm \text { SEM })\end{array}$ & $\begin{array}{c}\text { Hypoxia } \\
(\text { mean } \pm \text { SEM })\end{array}$ & p-value (t-test) \\
\hline $\mathbf{4}(\mathbf{a})$ & $0.919 \pm 0.039$ & $1.037 \pm 0.025$ & 0.030 \\
\hline $\mathbf{4}(\mathbf{b})$ & $0.932 \pm 0.026$ & $1.048 \pm 0.0325$ & 0.020 \\
\hline $\mathbf{4}(\mathbf{c})$ & $1.094 \pm 0.046$ & $0.910 \pm 0.065$ & 0.045 \\
\hline
\end{tabular}

Figure 5. Effect of hypoxia on the profile of a-globin isoforms in lung tissue

A. Detail of a reference 2D-DIGE gel from lung tissue samples, showing the spots identified as different a-globin forms $(4 a, b, c)$. B. Differences between tissue samples from normoxic and hypoxic animals with respect to the levels of the different a-globin forms, determined by the fluorescent intensity of the single spots. Globin forms that significantly increased under hypoxia are labeled in blue, and those with reduced amount, in red. 


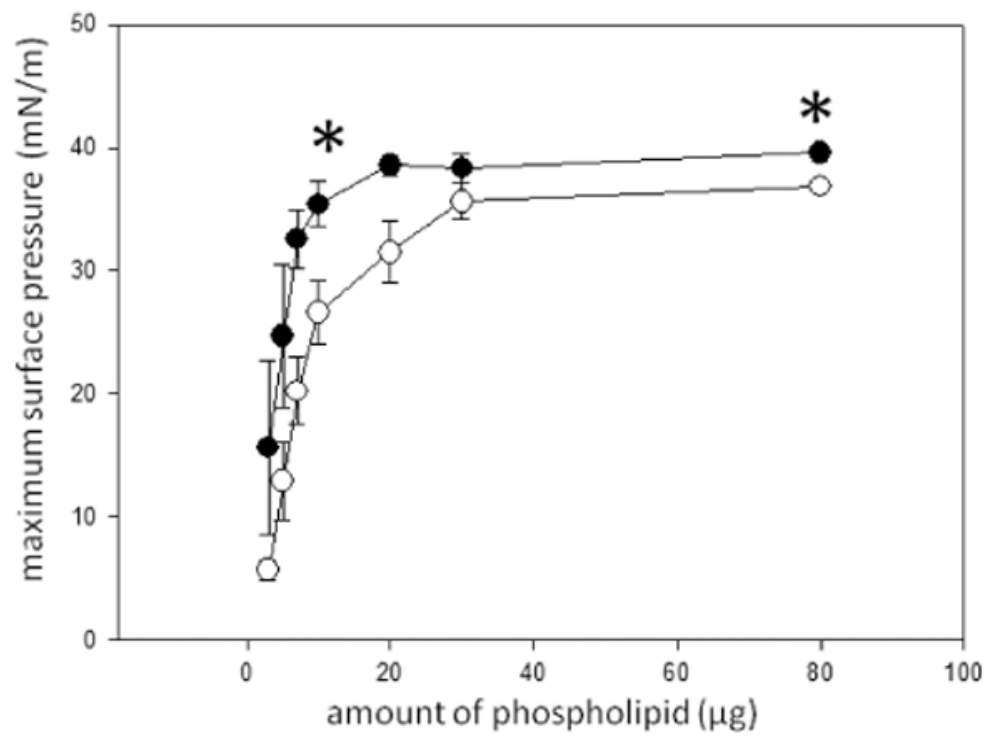

Figure 6. Effect of hypoxia on the interfacial spreading and adsorption ability of pulmonary surfactant

Plot of the maximum surface pressure achieved upon spreading of increasing amounts of pulmonary surfactant obtained from control normoxic (open circles) and hypoxic (closed circles). Data are presented as means with SEM $(n=3)$. Significant differences are shown as $*(\mathrm{p}<0.05)$. 
A. Bronchoalveolar lavages

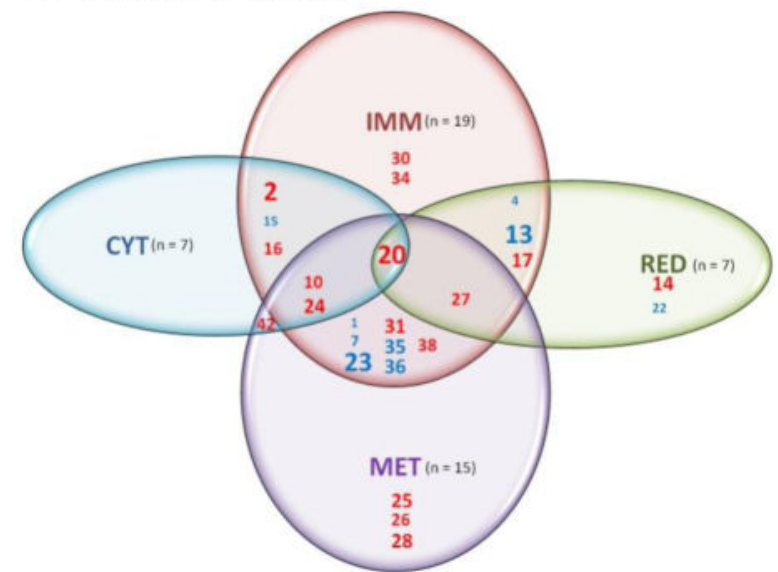

B. Lung tissue

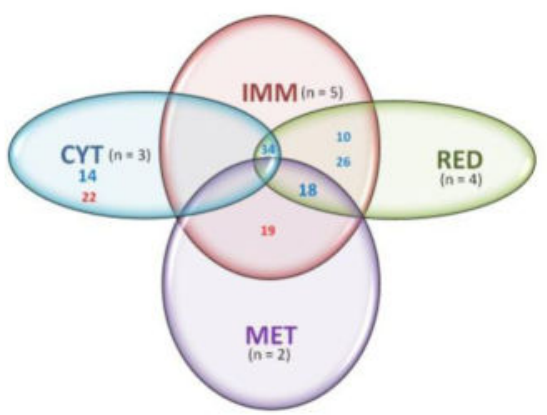

Figure 7. Profile of hypoxia-affected lung proteome

The diagrams represent the proteins exhibiting a significant content change as a consequence of hypoxia in their respective functional groups in BAL (A) and lung tissue (B). Group overlaps include proteins that are simultaneously involved in several of the functional groups. The numbers correspond to the proteins listed in supplementary Table 3 (reduced level in red, increased in blue). The size of the number font intended to reflect the extent of the change in protein level, according to the data in Tables 1 and 2. 
Table 1

Proteins in BAL whose levels are significantly altered as a consequence of exposure to hypoxia

\begin{tabular}{|c|c|c|}
\hline Number (see fig. 5) & Protein name & Percent change \\
\hline \multicolumn{3}{|l|}{ Significant increases } \\
\hline 13 & Ceruloplasmin & 134,7 \\
\hline 23 & Inter-alpha-inhibitor $\mathrm{H} 4$ heavy chain & 116.3 \\
\hline 36 & Serine protease inhibitor $2 \mathrm{~b}$ & 41,4 \\
\hline 35 & Serine protease inhibitor 1 & 30,3 \\
\hline 15 & Fetuin beta & 27,4 \\
\hline 4 & Albumin & 23,8 \\
\hline 1 & Aa2-001 & 22,4 \\
\hline 22 & Hemopexin & 22,2 \\
\hline \multicolumn{3}{|c|}{ Significant decreases } \\
\hline 20 & Haptoglobin & $-52,7$ \\
\hline 2 & Abhydrolase domain containing $14 \mathrm{~b}$ & $-50,7$ \\
\hline 24 & Lipocortin I & $-48,7$ \\
\hline 31 & Protein RAKc & $-46,8$ \\
\hline 14 & $\mathrm{Cu} / \mathrm{Zn}$ superoxide dismutase & $-45,5$ \\
\hline 25 & Non-metastatic cells 2, protein |NM23B) expressed in & $-44,6$ \\
\hline 28 & Phosphatidylethanolamine binding protein & $-44,2$ \\
\hline 17 & Glutathione S-transferase Y-b subunit(EC 2.5.1.18) & $-43,6$ \\
\hline 38 & Similar to SPI6 & $-39,3$ \\
\hline 34 & Selenium binding protein 1 & $-38,6$ \\
\hline 16 & Gelsolin & $-37,5$ \\
\hline 30 & Protein Plunc (Palate lung \& nasal epithelium clone protein) & $-36,1$ \\
\hline 10 & Annexin A5 (Lipocortin V) & $-35,6$ \\
\hline 27 & Peroxiredoxin 6 & $-35,2$ \\
\hline 42 & Tyrosine 3-monooxygenase/tryptophan 5-monooxygenaseacti,prot. & $-34,1$ \\
\hline 26 & Pcyt2 protein & $-31,1$ \\
\hline
\end{tabular}

To calculate the percent change of the proteins identified in BAL, the sum of the spot volumes for each protein in the control group was taken as $100 \%$. Therefore, a percent change of 50\% refers to an increase (positive values) or decrease (negative values) of the protein levels of 1.5 fold in the hypoxic with respect to the normoxic control group $(p<0.05)$. 
Table 2

Proteins in lung tissue whose levels are significantly altered as a consequence of exposure to hypoxia

\begin{tabular}{cll}
\hline $\begin{array}{c}\text { Number (see fig. 6) } \\
\text { Significant increases }\end{array}$ & Protein name & Percent change \\
18 & Grp75 (Mortalin) (Heat shock protein 9) & 31,4 \\
14 & Desmin & 31,1 \\
26 & Peroxiredoxin 4 & 26,6 \\
34 & Transferrin & 25,3 \\
10 & Calreticulin & 23,6 \\
Significant decreases & \\
22 & Lamin-A & $-18,5$ \\
19 & Guanine deaminase & -15.7 \\
\hline
\end{tabular}

Significant percent change of the proteins identified in lung homogenates. Negative values indicate a reduced level in the hypoxic group with respect to the normoxic group $(p<0.05)$. 